TRANSACTIONS OF THE

AMERICAN MATHEMATICAL SOCIETY

Volume 357, Number 5, Pages 1977-1994

S 0002-9947(04)03582-2

Article electronically published on October 7, 2004

\title{
THREEFOLDS WITH VANISHING HODGE COHOMOLOGY
}

\author{
JING ZHANG
}

\begin{abstract}
We consider algebraic manifolds $Y$ of dimension 3 over $\mathbb{C}$ with $H^{i}\left(Y, \Omega_{Y}^{j}\right)=0$ for all $j \geq 0$ and $i>0$. Let $X$ be a smooth completion of $Y$ with $D=X-Y$, an effective divisor on $X$ with normal crossings. If the $D$-dimension of $X$ is not zero, then $Y$ is a fibre space over a smooth affine curve $C$ (i.e., we have a surjective morphism from $Y$ to $C$ such that the general fibre is smooth and irreducible) such that every fibre satisfies the same vanishing condition. If an irreducible smooth fibre is not affine, then the Kodaira dimension of $X$ is $-\infty$ and the $D$-dimension of $X$ is 1 . We also discuss sufficient conditions from the behavior of fibres or higher direct images to guarantee the global vanishing of Hodge cohomology and the affineness of $Y$.
\end{abstract}

\section{INTRODUCTION}

Let $Y$ be a complex manifold with $H^{i}\left(Y, \Omega_{Y}^{j}\right)=0$ for all $j \geq 0$ and $i>0$. Then what is $Y$ ? Is $Y$ Stein? This is a question raised by Serre [Se]. Peternell [P] also asked the same question for schemes: If $Y$ is a smooth scheme of finite type over $\mathbb{C}$, is it affine? For the nonalgebraic case, in particular complex surfaces, see Peternell's paper $[\mathrm{P}$. Throughout this paper, we assume that $Y$ is an algebraic manifold, i.e., an irreducible nonsingular algebraic variety defined over $\mathbb{C}$. If $\operatorname{dim} Y=1$, then $Y$ is affine. If $\operatorname{dim} Y=2$, Mohan Kumar $\mathrm{Ku}$ classified it completely. It may not be affine and has three possibilities as follows:

(1) $Y$ is affine.

(2) Let $C$ be an elliptic curve and $E$ the unique nonsplit extension of $\mathcal{O}_{C}$ by itself. Let $X=\mathbb{P}_{C}(E)$ and $D$ the canonical section. Then $Y=X-D$.

(3) Let $X$ be a projective rational surface with an effective divisor $D=-K$ with $D^{2}=0$, let $\left.\mathcal{O}(D)\right|_{D}$ be nontorsion, and let the dual graph of $D$ be $\tilde{D}_{8}$ or $\tilde{E}_{8}$. Then $Y=X-D$.

If the surface $Y$ is not affine, then the Kodaira dimension of $X$ is $-\infty$ and the $D$-dimension is $0([\mathrm{Ku}]$, Lemma 1.8). In the second case, the canonical divisor $K_{X}=-2 D$ and $D$ is irreducible, so the logarithmic Kodaira dimension $\bar{\kappa}(Y)=$ $\kappa\left(D+K_{X}, X\right)=-\infty$; in the third case, $K_{X}=-D$ and $D$ is either $\tilde{D}_{8}$ or $\tilde{E}_{8}$. Let $D^{\prime}=\sum D_{i}$ be the reduced divisor, where the $D_{i}$ 's are the prime components of $D$; then $K_{X}+D^{\prime}$ is not effective. Therefore again $\bar{\kappa}(Y)=-\infty$ ([I3], [Ku], [Mi]). The surfaces in the first two cases are Stein. The third case is open. Since there exist

Received by the editors May 9, 2003 and, in revised form, November 21, 2003.

2000 Mathematics Subject Classification. Primary 14J30, 14B15, 14C20.

Key words and phrases. 3-folds, Hodge cohomology, local cohomology, fibration, higher direct images. 
Stein varieties which are not affine (see [H2], Serre gave the first example of this), the surfaces in the third case might be Stein.

If $\operatorname{dim} Y=3$, let us first fix our basic assumption (BA) as follows.

(BA) Let $Y$ be a smooth irreducible threefold, and let $X$ be a smooth completion (see Nagata $[\mathrm{N}]$ for the existence of $X$ ) such that $X-Y=\bigcup D_{i}$, the union of connected, distinct prime divisors on $X$. Let $D$ be an effective divisor supported in $\bigcup D_{i}$ with normal crossings [13. Suppose that the moving part of $|n D|$ is base point free (such a smooth completion of $Y$ always exists after further blowups), $H^{0}\left(X, \mathcal{O}_{X}(n D)\right) \neq \mathbb{C}$ for some $n$ and $Y$ contains no complete surfaces.

Proposition. Under the condition (BA), there is a smooth projective curve $\bar{C}$ and a smooth affine curve $C$ such that the following diagram commutes:

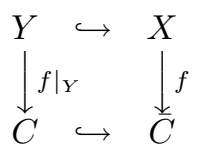

where $f$ is proper and surjective, every fibre of $f$ over $\bar{C}$ is connected, and the general fibre is smooth. Also, the general fibre of $\left.f\right|_{Y}$ is connected and smooth, and if the $D$-dimension of $X$ is no less than 2 , then we can set $\bar{C}$ to be $\mathbb{P}^{1}$.

Our main results are the following.

Theorem A. If $H^{i}\left(Y, \Omega_{Y}^{j}\right)=0$ for all $j \geq 0$ and $i>0$ and $H^{0}\left(Y, \mathcal{O}_{Y}\right) \neq \mathbb{C}$, then for the above $f$, we have:

(1) Every fibre $S$ of $\left.f\right|_{Y}$ over $C$ satisfies the same vanishing condition, i.e., $H^{i}\left(S, \Omega_{S}^{j}\right)=0$.

(2) If there is a smooth fibre $X_{y_{0}}$ over $y_{0} \in \bar{C}$ such that $\left.X_{y_{0}}\right|_{Y}=S_{0}$ is not affine, then the Kodaira dimension of $X$ is $-\infty$ and the D-dimension of $X$ is 1 .

(3) If one fibre $S$ of $\left.f\right|_{Y}$ over $C$ is not affine, then $Y$ is not affine. $Y$ is affine if and only if for every coherent sheaf $F$ on $X$

$$
h^{1}\left(X, \lim _{\vec{n}} F \otimes \mathcal{O}(n D)\right)<\infty .
$$

Conversely, in the above diagram, let $F_{n}=\Omega_{X}^{j} \otimes \mathcal{O}(n D)$ or $F_{n}=\Omega_{X}^{j}(\log D) \otimes$ $\mathcal{O}(n D)$, where $\Omega_{X}^{j}(\log D)$ is the sheaf of logarithmic $j$-forms on $Y$ (I1], [12], [13]). Then we have

Theorem B. If the higher direct images satisfy

$$
\lim _{\vec{n}} R^{1} f_{*} F_{n}=\lim _{\vec{n}} R^{2} f_{*} F_{n}=0,
$$

or for every point $y \in C$, if $D_{y}=X_{y} \cap D$ is a curve, and

$$
\lim _{\vec{n}} H^{2}\left(X_{y}, F_{n, y}\right)=0, \quad \lim _{\vec{n}} R^{1} f_{*} F_{n}=0,
$$

where $F_{n, y}=\left.F_{n}\right|_{X_{y}}$, then $H^{i}\left(Y, \Omega_{Y}^{j}\right)=0$ for all $j \geq 0$ and $i>0$.

Similar results for the affineness of $Y$ can be obtained. We will discuss it in Section 3. From Theorem $A$ and the surface cases (2) and (3) which are not affine, we know that a threefold with the vanishing Hodge cohomology is not necessarily affine. If it is affine, then of course it is Stein. In the surface case, if $Y$ is not affine, the Kodaira dimension of $X$ is unique. Is it still true for threefolds? When 
there are nonconstant regular functions on the threefold $Y$, by Theorem $\mathrm{A}(1)$ and Mohan Kumar's classification, there are three different types of smooth fibres. The question reduces to two questions. The first question is: given a smooth variety $Y$ with $H^{i}\left(Y, \Omega_{Y}^{j}\right)=0$ for all $j \geq 0, i>0$ and a surjective morphism from $Y$ to a smooth affine curve $C$ such that every (or general) fibre is affine, then is $Y$ affine? Generally it is not true without restriction on Hodge cohomology. Under our cohomology restrictions, if $Y$ is a surface, then it is true (Lemma 1.8, $[\mathrm{Ku}]$ ). The second question is the invariance of plurigenera. If one fibre $S_{0}$ is not affine, then is the Kodaira dimension of $X_{t}$ a constant in an open neighborhood of 0 ? Iitaka conjectured that in a smooth family the $m$ th plurigenus is constant. He proved it for the surface case ([I4], [I5]). Nakayama proved that the conjecture follows from the minimal model conjecture and the abundance conjecture ([Na1, [Na3]). Siu proved it if the generic fibre is of general type Si2. Kawamata extended Siu's result to fibres with canonical singularities $\mathrm{Ka} 4$. In our case, some isolated fibres may be singular or reducible or both. We cannot therefore apply these results.

Now let $Y$ and $X$ be as in Theorem A. We are sure that the Kodaira dimension of $X$ can be $-\infty$ and the $D$-dimension can be 1 (Theorem 7). Our motivation here is to see the global picture from the fibre, i.e., if every fibre or general fibre has vanishing Hodge cohomology, then is it still true for $Y$ ? We can prove that the direct limit of second direct images vanishes, thus $H^{2}\left(Y, \Omega_{Y}^{j}\right)=0$. But the direct limit of the first direct images might be supported at finitely many points. We do not know how to deal with these points and whether the first direct image sheaves are locally free or not. In fact, by a result of Goodman and Hartshorne (Lemma 4), we only need the local freeness on $C$. If it is true, then the direct limit is zero and therefore we also have $H^{1}\left(Y, \Omega_{Y}^{j}\right)=0$. Hence we can get an equivalent condition for vanishing Hodge cohomology of $Y$.

Mohan Kumar's proof in the surface case heavily depends on the following two facts. By his Lemma 1.10, any line bundle $L$ on the carefully chosen divisor $D$ with degree zero when restricted to each component of $D$ has the following property: $H^{0}(L) \neq 0$ if and only if $L \cong \mathcal{O}_{D}$. Thus if $L=\mathcal{O}_{D}(D)$, then it satisfies all these conditions by the choice of $D$; therefore, it is either torsion or nontorsion. This is why $Y$ has only two possibilities if it is not affine. We do not know of a similar result when the dimension is 3 . The second fact is the Zariski decomposition of $D$. He used it to compute intersection numbers and $h^{0}\left(X, \mathcal{O}_{X}(m D)\right)=1$ for every nonnegative $m$. But in threefold, we do not always have Zariski decomposition (see [C]; for recent progress, see [Na2]). To understand $Y$ and $X$, we have to use a different approach. We first construct a proper and surjective morphism from $X$ to a smooth curve. This is the place where we need the condition that the $D$ dimension of $X$ is not 0 . Notice that we cannot use any other divisor on $X$ to define our map. Otherwise, we have no control of the cohomology and the image of $Y$, so we cannot use our assumption. This says that we can only change the boundary $D$, but cannot change $Y$. This is why we cannot use Iitaka's fibration and Mori's construction. So in order to use Iitaka's fibration, we must assume $\kappa(X) \geq 0$, which is not true in our case; but we can use Iitaka's $C_{n}$ conjecture ([Ka2, [V]).

The content of this paper is divided into three parts. In the first section, we will present some basic lemmas. We borrow the idea from the surface case, i.e., from $\mathrm{Ku}, \mathrm{P}$. The second section contains the construction of the fibre space. We will prove our main theorems in the third section and give an example. Our basic tools 
are Grothendieck's local cohomology theory and classification theory developed by the Japanese school of algebraic geometry.

Convention. Unless otherwise explicitly mentioned, we always use Zariski topology, i.e., an open set means a Zariski open set.

\section{Preliminary Lemmas}

Lemma 1. Let $Y$ be an irreducible smooth threefold with $H^{i}\left(Y, \Omega_{Y}^{j}\right)=0$ for every $j \geq 0$ and $i>0$. Let $X$ be any smooth completion of $Y$. Then $X-Y$ has no isolated points.

Proof. If $P$ is an isolated point of $X-Y$, let $Y^{\prime}=Y \cup\{P\}$. Then $Y^{\prime}$ is a scheme and we have the exact sequence of local cohomology

$$
0=H^{2}\left(Y, \mathcal{O}_{Y}\right) \longrightarrow H_{\{P\}}^{3}\left(\mathcal{O}_{Y^{\prime}}\right) \longrightarrow H^{3}\left(Y^{\prime}, \mathcal{O}_{Y^{\prime}}\right)=0 .
$$

The last term is zero since $Y^{\prime}$ is not complete; but

$$
H_{\{P\}}^{3}\left(\mathcal{O}_{Y^{\prime}}\right) \cong \lim _{\vec{n}} \operatorname{Ext} t_{\mathcal{O}_{X}}^{3}\left(\mathcal{O}_{n P}, \mathcal{O}_{Y^{\prime}}\right) \neq 0
$$

where $\mathcal{O}_{n P}=\mathcal{O}_{X} / \mathcal{M}^{n}, \mathcal{M}$ is the ideal sheaf of $P$. To see this, write the short exact sequence

$$
0 \longrightarrow \mathcal{M}^{n} / \mathcal{M}^{n+1} \longrightarrow \mathcal{O}_{(n+1) P} \longrightarrow \mathcal{O}_{n P} \longrightarrow 0
$$

Then, since $\operatorname{Ext}_{\mathcal{O}_{X}}^{2}\left(\mathcal{M}^{n} / \mathcal{M}^{n+1}, \mathcal{O}_{Y^{\prime}}\right)=0$, we have

$$
\begin{aligned}
0 \longrightarrow \operatorname{Ext}_{\mathcal{O}_{X}}^{3}\left(\mathcal{O}_{n P}, \mathcal{O}_{Y^{\prime}}\right) & \longrightarrow \operatorname{Ext}_{\mathcal{O}_{X}}^{3}\left(\mathcal{O}_{(n+1) P}, \mathcal{O}_{Y^{\prime}}\right) \\
& \longrightarrow \operatorname{Ext}_{\mathcal{O}_{X}}^{3}\left(\mathcal{M}^{n} / \mathcal{M}^{n+1}, \mathcal{O}_{Y^{\prime}}\right) \longrightarrow 0
\end{aligned}
$$

If $\operatorname{Ext}_{\mathcal{O}_{X}}^{3}\left(\mathcal{M}^{n} / \mathcal{M}^{n+1}, \mathcal{O}_{Y^{\prime}}\right) \neq 0$, then dimension of $\operatorname{Ext}_{\mathcal{O}_{X}}^{3}\left(\mathcal{O}_{n P}, \mathcal{O}_{Y^{\prime}}\right) \longrightarrow \infty$ as $n \longrightarrow \infty$. Thus $H_{\{P\}}^{3}\left(\mathcal{O}_{Y^{\prime}}\right) \neq 0$. For some suitable $m$ determined by $n$, we have

$$
\mathcal{M}^{n} / \mathcal{M}^{n+1}=\left(\mathcal{O}_{P} / \mathcal{M}\right)^{m} .
$$

Therefore,

$$
\operatorname{Ext}_{\mathcal{O}_{X}}^{3}\left(\mathcal{M}^{n} / \mathcal{M}^{n+1}, \mathcal{O}_{Y^{\prime}}\right)=\bigoplus \operatorname{Ext}^{3}\left(\mathcal{O}_{P} / \mathcal{M}, \mathcal{O}_{Y^{\prime}}\right)=\bigoplus \operatorname{Ext}^{3}\left(\mathbb{C}(P), \mathcal{O}_{Y^{\prime}}\right)
$$

Choose local coordinates such that $P=\{x=y=z=0\}, x, y, z \in \mathcal{O}_{U}, U$ is a neighborhood of $P$. Then

$$
0 \longrightarrow \mathcal{O}_{U} \longrightarrow \mathcal{O}_{U}^{3} \longrightarrow \mathcal{O}_{U}^{3} \longrightarrow \mathcal{O}_{U} \longrightarrow \mathbb{C}(P) \longrightarrow 0 .
$$

Moreover,

$$
\mathcal{E} x t^{i}\left(\mathbb{C}(P), \mathcal{O}_{U}\right)= \begin{cases}0 & \text { if } i \neq 3 \\ \mathbb{C}(P) & \text { if } i=3\end{cases}
$$

Finally, we can compute

$$
\operatorname{Ext}^{3}\left(\mathbb{C}(P), \mathcal{O}_{Y^{\prime}}\right)=H^{0}\left(\mathcal{E} x t^{3}\left(\mathbb{C}(P), \mathcal{O}_{U}\right)\right)=\mathbb{C}(P) \neq 0 .
$$

Lemma 2. Under the conditions of Lemma $1, X-Y=\bigcup Z_{i}$ is connected, where the $Z_{i}$ 's are irreducible components. 
Proof. If $X-Y=Z$ is not connected, write $Z=Z_{1}+Z_{2}, Z_{1} \cap Z_{2}=\emptyset$. We have a long exact sequence of local cohomology:

$$
0=H^{2}\left(Y, \Omega_{Y}^{3}\right) \longrightarrow H_{Z}^{3}\left(X, \Omega_{X}^{3}\right) \longrightarrow H^{3}\left(X, \Omega_{X}^{3}\right) \longrightarrow H^{3}\left(Y, \Omega_{Y}^{3}\right)=0 .
$$

So $H_{Z}^{3}\left(X, \Omega_{X}^{3}\right)=H^{3}\left(X, \Omega_{X}^{3}\right)=\mathbb{C}$ by Serre duality; but by the Mayer-Vietoris sequence,

$$
H_{Z}^{3}\left(X, \Omega_{X}^{3}\right) \cong H_{Z_{1}}^{3}\left(X, \Omega_{X}^{3}\right) \oplus H_{Z_{2}}^{3}\left(X, \Omega_{X}^{3}\right) .
$$

Both summands are at least one-dimensional since

$$
H_{Z_{i}}^{3}\left(X, \Omega_{X}^{3}\right) \longrightarrow H^{3}\left(X, \Omega_{X}^{3}\right)=\mathbb{C} \longrightarrow H^{3}\left(X-Z_{i}, \Omega_{X}^{3}\right)=0 .
$$

This is a contradiction.

Lemma 3. Let $X, Y$ be as above. Then $Y$ contains no complete surfaces.

Proof. If $S$ is a complete, irreducible surface in $Y$, then we have the short exact sequence

$$
0 \longrightarrow A \longrightarrow \Omega_{Y}^{2} \longrightarrow \Omega_{S}^{2} \longrightarrow 0
$$

where $A$ is the kernel. Since $\Omega_{Y}^{2}$ and $\Omega_{S}^{2}$ are coherent, $A$ is coherent. For any abelian sheaf $\mathcal{F}$ on $X$, we have the long exact sequence

$$
\cdots \longrightarrow H_{Z}^{3}(X, \mathcal{F}) \longrightarrow H^{3}(X, \mathcal{F}) \longrightarrow H^{3}(Y, \mathcal{F}) \longrightarrow 0 .
$$

By formal duality []ㅡ,

$$
H^{0}(\hat{X}, \hat{\mathcal{G}})=H_{Z}^{3}(X, \mathcal{H})^{*}
$$

where $\mathcal{G}=\mathcal{H o m}_{\mathcal{O}_{X}}(\mathcal{F}, \omega), \omega=\Omega_{X}^{3}$ and $\mathcal{H}=\mathcal{H} m_{\mathcal{O}_{X}}(\mathcal{G}, \omega)$. If $\mathcal{F}$ is locally free, then $\mathcal{H}=F$. So we have

$$
H^{0}(\hat{X}, \hat{\mathcal{G}})=H_{Z}^{3}(X, \mathcal{F})^{*}
$$

but $H^{0}(X, \mathcal{G}) \longrightarrow H^{0}(\hat{X}, \hat{\mathcal{G}})$ is injective, by Serre duality, $H_{Z}^{3}(X, \mathcal{F}) \longrightarrow H^{3}(X, \mathcal{F})$ is surjective. So $H^{3}(Y, \mathcal{F})=0$ for any locally free sheaf $\mathcal{F}$. Then for any coherent sheaf $\mathcal{F}, H^{3}(Y, \mathcal{F})=0$ since we have the short exact sequence

$$
0 \longrightarrow B \longrightarrow \mathcal{F}^{\prime} \longrightarrow \mathcal{F} \longrightarrow 0
$$

where $\mathcal{F}^{\prime}$ is locally free. In particular, $H^{3}(Y, A)=0$. From

$$
0=H^{2}\left(Y, \Omega_{Y}^{2}\right) \longrightarrow H^{2}\left(\Omega_{S}^{2}\right) \longrightarrow H^{3}(Y, A)=0
$$

we have $H^{2}\left(\Omega_{S}^{2}\right)=0$, which is a contradiction $([\mathrm{AK}])$.

Remark 1. Our proof is algebraic. In analytic category, we can use Siu's theorem Si1] to get $H^{3}(Y, F)=0$ for any analytic sheaf $F$ since $Y$ is not compact by Serre duality. Then we can use Norguet and Siu's result ([NS], $[\mathrm{P}]$ ). It says that if a complex manifold $Y$ contains a compact analytic subvariety of dimension $q$ and for every coherent sheaf $F$ on $Y, H^{q+1}(Y, F)=0$, then $H^{q}\left(Y, \Omega^{q}\right) \neq 0$.

By the above lemmas, we know that for any smooth completion $X$ of $Y$, the dimension of the boundary $X-Y$ is not zero. By suitable blowing ups, we may assume they satisfy the basic assumption (BA). So the $D$-dimension of such $X$ makes sense. We put a lemma after Theorem 1 for logical correctness. It says that if the $D$-dimension of $X$ is not zero, then $Y$ contains no complete curves. 
Lemma 4 (Goodman, Hartshorne). Let $V$ be a scheme and $D$ an effective Cartier divisor on $V$. Let $U=V-S u p p D$, and let $F$ be any coherent sheaf on $V$. Then for every $i \geq 0$,

$$
\lim _{\vec{n}} H^{i}(V, F \otimes \mathcal{O}(n D)) \cong H^{i}\left(U,\left.F\right|_{U}\right)
$$

\section{Construction of a proper, surjective morphism from $X$ to $\bar{C}$}

If $H^{0}\left(X, \mathcal{O}_{X}(n D)\right) \neq \mathbb{C}$, let $\xi$ be a nonconstant, irreducible element (which means that it cannot be written as a product of two nonconstant elements) in $H^{0}\left(X, \mathcal{O}_{X}(n D)\right)$. Then it defines a rational map

$$
\xi: X \rightarrow \mathbb{P}^{1},
$$

with poles in $D$. When restricted to $Y$, it is a morphism

$$
\left.\xi\right|_{Y}: Y \longrightarrow \mathbb{A}^{1} \text {. }
$$

Let $U=\xi(Y)$, the image of $Y$ under $\xi$. By Hironaka's elimination of indeterminacy, there is a smooth projective variety $\tilde{X}$, such that the morphism $\sigma: \tilde{X} \rightarrow X$ is a composite of finitely many monoidal transformations which is isomorphic when restricted to $Y$, i.e., $Y$ is fixed and $g=\xi \circ \sigma: \tilde{X} \rightarrow \mathbb{P}^{1}$ is proper and surjective. Replace $X$ by $\tilde{X}$. Since $\left.g\right|_{Y}=\xi$, we have the following commutative diagram:

$$
\begin{array}{cccc}
Y & \hookrightarrow & X \\
\mid \downarrow & & & \\
U & & & \downarrow \\
U & & \mathbb{P}^{1} .
\end{array}
$$

To guarantee the connectedness of fibres, we can use Stein factorization. Let $f$ : $X \rightarrow \bar{C}$ be a proper surjective morphism, and let $h: \bar{C} \rightarrow \mathbb{P}^{1}$ be a finite ramified covering such that $g$ is the composition of these two maps, i.e., $g=h \circ f$. Let $C=f(Y)$. Then we have the commutative diagram

$$
\begin{aligned}
& Y \hookrightarrow X \\
& \left.\downarrow f\right|_{Y} \\
& \stackrel{\downarrow}{C} \hookrightarrow \stackrel{\downarrow}{C},
\end{aligned}
$$

where $f$ is proper and surjective and every fibre of $f$ is connected. Moreover, $C$ and $\bar{C}$ are smooth.

Now consider the image of $D$ under $f$. If $f(D)$ is a point, then $Y$ contains complete surfaces, so $f(D)=\bar{C}$. Since both $X$ and $\bar{C}$ are irreducible and projective, every fibre of $f$ over $\bar{C}$ has dimension at least 2 ( $\underline{\mathrm{Sh}}$, Chapter 1 , section 6.3, Theorem 7) but cannot be 3 ( $[\mathrm{Sh}]$, Chapter 1, section 6.1 , Theorem 1), that is, every fibre has dimension 2. By the second Bertini theorem ( $\underline{\mathrm{Sh}}]$, Chapter 2, section 6.2), there is an open set $U \subset \bar{C}$ such that every fibre $f^{-1}(P)$ for every point $P$ in $U$ is smooth.

Since $f(D)=\bar{C}$, there is a component $D_{i}$ of $D$, such that $f\left(D_{i}\right)=\bar{C}$. But some components of $D$ may have points as images. Removing these finitely many points from $C$, for a general point $P$ in $C$, the inverse image $\bar{S}=f^{-1}(P)$ is an irreducible surface such that $\emptyset \neq D_{i} \cap \bar{S} \subset D \cap \bar{S}$. By irreducibility of the general fibre, $D \cap \bar{S}$ is a curve on $\bar{S}$ for general $P$. Removing this curve, the surface $S=\bar{S}-D=\bar{S} \cap Y$ is irreducible. So the general fibre of $\left.f\right|_{Y}$ over $C$ is smooth and irreducible (thus connected). 
By our construction, $f_{*} \mathcal{O}_{X}=\mathcal{O}_{\bar{C}}([\mathrm{U} 2]$, Proposition 1.13). But we do not know what the curve $\bar{C}$ is. If the $D$-dimension $\kappa(D) \geq 2$, then we can set $\bar{C}$ to be $\mathbb{P}^{1}$. The construction of a rational map from $X$ to $\mathbb{P}^{1}$ is due to Ueno ([U2], page 46).

Choose two algebraically independent rational functions $\eta_{1}$ and $\eta_{2}$ in $\mathbb{C}(X)$. By Zariski's lemma ( $[\mathrm{HP}$, Chapter $\mathrm{X}$, section 13 , Theorem 1, page 78$)$, there exists a constant $d$ such that the field $\mathbb{C}\left(\eta_{1}+d \eta_{2}\right)$ is algebraically closed in $\mathbb{C}(X)$. Define a rational map $f$ from $X$ to $\mathbb{P}^{1}$ by sending points $x$ in $X$ to $\left(1, \eta_{1}(x)+d \eta_{2}(x)\right)$ in $\mathbb{P}^{1}$. We can choose $\eta_{1}$ and $\eta_{2}$, such that $\eta_{1}+d \eta_{2}$ only has poles in $D$ ([U2], Lemma 4.20.3), that is, when restricted to $Y, f$ is a morphism. Then by our previous argument, we have the diagram

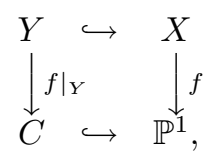

where $f$ and $\left.f\right|_{Y}$ satisfy the same properties as before.

Proposition. Under the condition (BA), there is a smooth projective curve $\bar{C}$ and a smooth, affine curve $C$ such that the following diagram commutes:

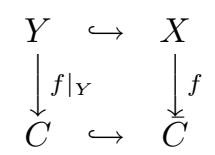

where $f$ is proper and surjective, every fibre of $f$ over $\bar{C}$ is connected, and the general fibre of $f$ is smooth. Also, the general fibre of $\left.f\right|_{Y}$ is connected and smooth. Moreover, if the D-dimension of $X$ is no less than 2 , then we can set $\bar{C}$ to be $\mathbb{P}^{1}$.

Remark 2. By [1]], page 79, for the general fibre $X_{y}=f^{-1}(y),\left.D\right|_{X_{y}}=D_{y}$ is a divisor of $X_{y}$ with normal crossings if $D$ is a divisor with normal crossings.

\section{Structure of $Y$ with $h^{0}\left(X, \mathcal{O}_{X}(n D)\right)>1$}

In the diagram of the proposition, since $\bar{C}$ is smooth, $f$ is flat.

Theorem 1. If $Y$ is a smooth threefold with $H^{i}\left(Y, \Omega_{Y}^{j}\right)=0$ for all $j \geq 0$ and $i>0$ and $H^{0}\left(X, \mathcal{O}_{X}(n D)\right) \neq \mathbb{C}$ for some $n$, then in the construction of the proposition, for every fibre $S$ of $\left.f\right|_{Y}$ over $C, H^{i}\left(S,\left.\Omega_{Y}^{j}\right|_{S}\right)=0$; therefore $H^{i}\left(S, \Omega_{S}^{j}\right)=0$ for all $j \geq 0$ and $i>0$.

Proof. $Y$ has no complete surfaces by Lemma 1. Thus the condition of the proposition is satisfied. For any point $P$ on $C$, let $g$ be an element of $\Gamma\left(C, \mathcal{O}_{C}\right)$ such that the divisor defined by $g$ is $Q=\operatorname{div} g=P+Q_{1}+\cdots+Q_{r}, P \neq Q_{i}$ for every $i$. Then

$$
f^{-1}(Q)=S_{Q}=S \cup S_{1} \cup \cdots \cup S_{r}
$$

where $S$ is the fibre over $P$ and $S_{i}$ is the fibre over $Q_{i}$. From the short exact sequence

$$
0 \longrightarrow \mathcal{O}_{Y} \longrightarrow \mathcal{O}_{Y} \longrightarrow \mathcal{O}_{S_{Q}} \longrightarrow 0
$$

where the first map is defined by $g$, we have

$$
H^{i}\left(S_{Q}, \mathcal{O}_{S_{Q}}\right)=0
$$

for every $i>0$. Similarly, from the short exact sequence

$$
\left.0 \longrightarrow \Omega_{Y}^{j} \longrightarrow \Omega_{Y}^{j} \longrightarrow \Omega_{Y}^{j}\right|_{S_{Q}} \longrightarrow 0
$$


where the first map is still defined by $g$, we have

$$
H^{i}\left(S_{Q},\left.\Omega_{Y}^{j}\right|_{S_{Q}}\right)=0 \text {. }
$$

By the Mayer-Vietoris sequence, we have

$$
H^{i}\left(S,\left.\Omega_{Y}^{j}\right|_{S}\right)=0 .
$$

In particular, $H^{i}\left(S, \mathcal{O}_{S}\right)=0$. From the exact sequence

$$
\left.0 \longrightarrow A \longrightarrow \Omega_{Y}^{j}\right|_{S} \longrightarrow \Omega_{S}^{j} \longrightarrow 0
$$

we have $H^{i}\left(S, \Omega_{S}^{j}\right)=0$ for every $i>0$ and $j \geq 0$ since $H^{2}(S, A)=0$ for the coherent sheaf $A([\mathrm{H} 3],[\mathrm{K}]])$.

Remark 3. If $H^{i}\left(Y, \Omega_{Y}^{j}\right)=0$ for every $i>0$ and $j \geq 0$, and $H^{0}\left(X, \mathcal{O}_{X}(n D)\right) \neq \mathbb{C}$ for some $n$, then $Y$ contains no complete curves. In fact, if $E$ is such a curve in $Y$, then its image under $\left.f\right|_{Y}$ is a point $P$ on $C$, so $E$ is contained in the fibre $S$ of $\left.f\right|_{Y}$ over $P$ also contained in $f^{-1}(P)=X_{P}$ in $X$. Write $X_{P}=X_{P}^{\prime}+D^{\prime}$ where $D^{\prime}$ is a divisor contained in $D$ and $X_{P}^{\prime}$ intersects $Y$ with the surface $S$ in $Y$, i.e., $S=Y \cap X_{P}^{\prime}$, and $X_{P}^{\prime} \cap D$ is a curve. Then $H^{i}\left(S, \Omega_{S}^{j}\right)=0$ for every $i>0$ and $j \geq 0$ by Theorem 1. This implies that $S$ is not complete [AK]. If there is a complete curve $Z$ in $S$, then $H^{1}\left(Z, \Omega_{Z}^{1}\right) \neq 0$, but

$$
0 \longrightarrow A \longrightarrow \Omega_{S}^{1} \longrightarrow \Omega_{Z}^{1} \longrightarrow 0
$$

and $\left.H^{2}(S, A)=0([\mathrm{H} 3],[\mathrm{K}]]\right)$. This is a contradiction. So we have shown

Lemma 5. Let $Y$ be a smooth threefold with $H^{i}\left(Y, \Omega_{Y}^{j}\right)=0$ for all $j \geq 0$ and $i>0$ and $H^{0}\left(Y, \mathcal{O}_{Y}\right) \neq \mathbb{C}$. Then $Y$ contains no complete curves.

Now consider the sheaves $\Omega_{X}^{j}$ and $\Omega_{X}^{j}(\log D)$. Let $F_{n}=\Omega_{X}^{j} \otimes \mathcal{O}(n D)$ or $F_{n}=$ $\Omega_{X}^{j}(\log D) \otimes \mathcal{O}(n D)$. Then $F_{n}$ is flat over $\bar{C}$ since it is locally free on $X$ and $\bar{C}$ is smooth. If $H^{i}\left(Y, \Omega_{Y}^{j}\right)=0$, then for every fibre $S=f_{\mid Y}^{-1}(y)=X_{y} \cap Y, y \in C$, $H^{i}\left(S,\left.\Omega_{Y}^{j}\right|_{S}\right)=0$ for all $j \geq 0$ and $i>0$. If $X_{y}$ is irreducible, then by Lemma 4 , since $\left.F_{n}\right|_{S}=\left.\Omega_{Y}^{j}\right|_{S}$ and $\left.D\right|_{X_{y}}=D_{y}$ is a divisor on $X_{y}$, we have

$$
\lim _{\vec{n}} H^{i}\left(X_{y}, F_{n, y}\right)=0
$$

where $F_{n, y}=\left.F_{n}\right|_{X_{y}}$. If the point $y$ lies in $\bar{C} \backslash C$, or the fibre $X_{y}$ is not irreducible, then what will happen? Is the direct limit still zero? Theorem 2 is our answer.

Theorem 2. Under the conditions of Theorem 1 , for every point $y$ in $\bar{C}$, we have

$$
\lim _{\vec{n}} H^{i}\left(X_{y}, F_{n, y}\right)=0 \text {. }
$$

Proof. If $y$ is contained in $C$ and the fibre $X_{y}$ in $X$ is irreducible, we are done. First let $y \in \bar{C} \backslash C, E=f^{-1}(y)$. We have the short exact sequence

$$
0 \longrightarrow \mathcal{O}_{X}(-E) \longrightarrow \mathcal{O}_{X} \longrightarrow \mathcal{O}_{E} \longrightarrow 0
$$

Tensoring with $F_{n}$, we have

$$
\left.0 \longrightarrow F_{n}(-E) \longrightarrow F_{n} \longrightarrow F_{n}\right|_{E} \longrightarrow 0
$$

If

$$
\lim _{\vec{n}} H^{i}\left(X, F_{n}(-E)\right)=0
$$


since we have

$$
\lim _{\vec{n}} H^{i}\left(X, F_{n}\right)=0
$$

then writing the long exact sequence, we get our claim.

For any fixed $n$, there is a suitable $l$, such that the map

$$
\alpha_{2}: \quad H^{i}\left(X, F_{n}\right) \longrightarrow H^{i}\left(X, F_{n+l-1}\right)
$$

is zero. For this $n$ and $l$, we have a map

$$
H^{i}\left(X, F_{n}(-E)\right) \stackrel{\alpha}{\longrightarrow} H^{i}\left(X, F_{n+l}(-E)\right) .
$$

$E$ is a component of $D$ (may not be prime) so the map $\alpha$ can be factored through three maps as follows:

$$
H^{i}\left(X, F_{n}(-E)\right) \stackrel{\alpha_{1}}{\longrightarrow} H^{i}\left(X, F_{n}\right) \stackrel{\alpha_{2}}{\longrightarrow} H^{i}\left(X, F_{n+l-1}\right) \stackrel{\alpha_{3}}{\longrightarrow} H^{i}\left(X, F_{n+l}(-E)\right) .
$$

Since $\alpha_{2}=0$, and $\alpha=\alpha_{3} \circ \alpha_{2} \circ \alpha_{1}$, we have $\alpha=0$, i.e, the direct limit we want is zero. The map $\alpha_{3}$ is the natural map corresponding to the map $F_{n+l-1} \rightarrow$ $F_{n+l-1} \otimes \mathcal{O}(D-E)$.

If $y$ is a point in $C$ and the fibre $X_{y}$ is not irreducible, write $X_{y}=X_{y}^{\prime}+D^{\prime}$ where $D^{\prime}$ is a divisor contained in $D, X_{y}^{\prime}$ intersects $Y$ with a surface $S$, and $X_{y}^{\prime} \cap D$ is a curve. Then $S$ is the fibre of $\left.f\right|_{Y}$ over $y$ in $Y$. $S$ may not be irreducible, however, $X_{y}^{\prime} \backslash S$ is a divisor on $X_{y}^{\prime}$. By Theorem 1 and Lemma 4, for every $i>0$,

$$
\lim _{\vec{n}} H^{i}\left(X_{y}^{\prime},\left.F_{n}\right|_{X_{y}^{\prime}}\right)=0 .
$$

From the short exact sequence

$$
\left.0 \longrightarrow F_{n}\left(-X_{y}^{\prime}\right) \longrightarrow F_{n} \longrightarrow F_{n}\right|_{X_{y}^{\prime}} \longrightarrow 0
$$

we have for $i+1=2,3$,

$$
\lim _{\vec{n}} H^{i+1}\left(X, F_{n}\left(-X_{y}^{\prime}\right)\right)=0
$$

Similar to the above argument about $H^{i}\left(X, F_{n}(-E)\right)$, we can see that

$$
\lim _{\vec{n}} H^{i}\left(X, F_{n}\left(-D^{\prime}\right)\right)=0
$$

For $i>0$, consider the map

$$
H^{i+1}\left(X, F_{n}\left(-X_{y}^{\prime}-D^{\prime}\right)\right) \stackrel{\beta}{\longrightarrow} H^{i+1}\left(X, F_{n+l}\left(-X_{y}^{\prime}-D^{\prime}\right)\right) .
$$

As before, it can be factored through three maps as follows:

$$
\begin{aligned}
H^{i+1}\left(X, F_{n}\left(-X_{y}^{\prime}-D^{\prime}\right)\right) & \stackrel{\beta_{1}}{\longrightarrow} H^{i+1}\left(X, F_{n}\left(-X_{y}^{\prime}\right)\right) \stackrel{\beta_{2}}{\longrightarrow} H^{i+1}\left(X, F_{n+l-1}\left(-X_{y}^{\prime}\right)\right) \\
& \stackrel{\beta_{3}}{\longrightarrow} H^{i+1}\left(X, F_{n+l}\left(-X_{y}^{\prime}-D^{\prime}\right)\right) .
\end{aligned}
$$

For every fixed $n$, we can choose $l$ such that the map $\beta_{2}$ is zero. Since $\beta=\beta_{3} \circ \beta_{2} \circ \beta_{1}$, $\beta=0$, i.e., for $i+1=2,3$,

$$
\lim _{\vec{n}} H^{i+1}\left(X, F_{n}\left(-X_{y}^{\prime}-D^{\prime}\right)\right)=0
$$

Again from the exact sequence

$$
\left.0 \longrightarrow F_{n}\left(-X_{y}^{\prime}-D^{\prime}\right) \longrightarrow F_{n} \longrightarrow F_{n}\right|_{X_{y}^{\prime}+D^{\prime}}=\left.F_{n}\right|_{X_{y}} \longrightarrow 0
$$

we get our claim. 
From Theorems 1 and 2 we know how the global vanishing cohomology controls the local (fibre) cohomology. How does the fibre behavior influence the global behavior? If every fibre $S$ of $\left.f\right|_{Y}$ in $Y$ over $y \in C$ satisfies $H^{i}\left(S,\left.\Omega_{Y}^{j}\right|_{S}\right)=0$, then does $H^{i}\left(Y, \Omega_{Y}^{j}\right)=0$ ? We will see that the second and third cohomology vanish but the first cohomology is a mystery. To guarantee its vanishing, we have to add a mild condition.

To see how the local fibre behavior influences the global behavior, the higher direct images $R^{i} f_{*} F_{n}$ are the link. They are coherent for all $i \geq 0$ by Grauert's theorem. Since $f$ is flat over $\bar{C}, h^{i}\left(X_{y}, F_{n, y}\right)=\operatorname{dim}_{\mathbb{C}} H^{i}\left(X_{y}, F_{n, y}\right)$ is an upper semicontinuous function on $\bar{C}$. Since $H^{4}\left(X_{y}, F_{n, y}\right)=H^{3}\left(X_{y}, F_{n, y}\right)=0$, by $\mathrm{Mu}$, Corollary $3, R^{3} f_{*} F_{n}=0$. This guarantees $H^{3}\left(Y, \Omega_{Y}^{j}\right)=0$ for every $j$, and for every point $y \in \bar{C}$,

$$
R^{2} f_{*} F_{n} \otimes \mathbb{C}(y) \cong H^{2}\left(X_{y}, F_{n, y}\right) .
$$

If we only consider the closed points $y$ on $\bar{C}, \mathbb{C}(y)=\mathbb{C}$, we have ([U2], Theorem 1.4)

$$
\left(R^{2} f_{*} F_{n}\right)_{y} \otimes \mathbb{C} \cong H^{2}\left(X_{y}, F_{n, y}\right),
$$

where $\left(R^{2} f_{*} F_{n}\right)_{y}$ is the stalk at $y$ and the tensor product is over $\mathcal{O}_{\bar{C}, y}$. So every stalk satisfies

$$
\lim _{\vec{n}}\left(R^{2} f_{*} F_{n}\right)_{y} / \mathcal{P}\left(R^{2} f_{*} F_{n}\right)_{y}=\lim _{\vec{n}} H^{2}\left(X_{y}, F_{n, y}\right)=0
$$

for every closed point $y$, where $\mathcal{P}$ is the maximal ideal of $\mathcal{O}_{y}$. This means that for every fixed $n$ and fixed $y$, there is an $l$ such that the map

$$
\phi: \quad\left(R^{2} f_{*} F_{n}\right)_{y} / \mathcal{P}\left(R^{2} f_{*} F_{n}\right)_{y} \longrightarrow\left(R^{2} f_{*} F_{n+l}\right)_{y} / \mathcal{P}\left(R^{2} f_{*} F_{n+l}\right)_{y}
$$

is zero. Choose an affine open neighborhood $U$ of $y$ in $\bar{C}$ such that $\left.R^{2} f_{*} F_{n}\right|_{U}=\tilde{M}$ and $\left.R^{2} f_{*} F_{n+l}\right|_{U}=\tilde{N}$, where $M$ and $N$ are finitely generated modules over $A=$ $\mathcal{O}(U)$. For every maximal ideal $\mathcal{P}$ of $\mathcal{O}(U)$, we have the commutative diagram

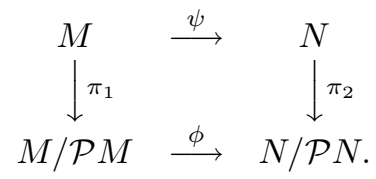

We can prove $\psi(M)=0$ if $D_{y}=D \cap X_{y}$ is a curve on the fibre $X_{y}$ for every $y \in C$. Therefore,

$$
\left.\lim _{\vec{n}} R^{2} f_{*} F_{n}\right|_{C}=0
$$

which means $H^{2}\left(Y, \Omega_{Y}^{j}\right)=0$ by Remark 5 . In fact, we can get a stronger result. From the exact sequence

$$
0 \longrightarrow \mathcal{O}(n D) \longrightarrow \mathcal{O}((n+1) D) \longrightarrow \mathcal{O}_{D}((n+1) D) \longrightarrow 0,
$$

tensoring with $F$ then with $\mathcal{O}_{X_{y}}$, we have

$$
\left.0 \longrightarrow F_{n, y} \longrightarrow F_{n+1, y} \longrightarrow F_{n+1, y}\right|_{D} \longrightarrow 0 .
$$

If $D_{y}$ is a curve, then $H^{2}\left(\left.F_{n+1, y}\right|_{D}\right)=H^{2}\left(F_{n+1, D_{y}}\right)=0$ for every $n$. So the map $H^{2}\left(F_{n, y}\right) \rightarrow H^{2}\left(F_{n+1, y}\right)$ is surjective for every $n$. But by Theorem 2 , for suitable $l$, the map $H^{2}\left(F_{n, y}\right) \rightarrow H^{2}\left(F_{n+l, y}\right)$ is zero. Thus there is an $n(y)$ depending on $y$ such that for every $n \geq n(y), H^{2}\left(F_{n, y}\right)=0$. Now fix some $y_{0}$ in $\bar{C}$ such that $H^{2}\left(F_{n, y_{0}}\right)=0$ for every $n \geq n\left(y_{0}\right)$ and there is an open neighborhood $U_{0}$ 
of $y_{0}$ in $\bar{C}$ such that $R^{2} f_{*} F_{n\left(y_{0}\right)}$ is locally free on $U_{0}$. Then $H^{2}\left(F_{n\left(y_{0}\right), y}\right)=0$ for every $y$ in $U_{0}$. So $H^{2}\left(F_{n, y}\right)=0$ for every $y$ in $U_{0}$ and every $n \geq n\left(y_{0}\right)$. Let $C-U_{0}=\left\{y_{1}, y_{2}, \ldots, y_{m}\right\}$ and choose $n_{0}=\max \left(n\left(y_{0}\right), n\left(y_{1}\right), \ldots, n\left(y_{m}\right)\right)$. Then $H^{2}\left(X_{y}, F_{n, y}\right)=0$ for every $y \in C$ and every $n \geq n_{0}$. By the upper semi-continuity theorem, $\left(R^{2} f_{*} F_{n}\right)_{y} / \mathcal{P}\left(R^{2} f_{*} F_{n}\right)_{y}=0$ for all points $y$ in $C$. By Nakayama's lemma, $\left.R^{2} f_{*} F_{n}\right|_{C}=0$.

The sheaf $R^{1} f_{*} F_{n}$ is not so nice. For any fixed $n$, there is an open set $U_{n}$ in $\bar{C}$, such that it is locally free on $U_{n}$. Let $U_{n}=\bar{C} \backslash A_{n}$, where $A_{n}$ is closed in $\bar{C}$, i.e., it consists of only finitely many points of $\bar{C}$. Since any complete metric space is a Baire space (in complex topology, every countable intersection of dense open sets in $\bar{C}$ is dense in $\bar{C}$ [B2], Chapter 9), $B=\bar{C} \backslash \bigcup A_{n}=\bigcap U_{n}$ is a dense (but we do not know if $B$ is open) subset of $\bar{C}$ in complex topology. Hence for every point $y$ on $B$, all stalks $\left(R^{1} f_{*} F_{n}\right)_{y}$ are locally free. Write $B$ as a union of connected subsets $B_{m}, B=\bigcup B_{m}$. Then there is one $B_{m}$, such that $B_{m}$ is dense in $\bar{C}$ and connected in complex topology. So we may assume that $B$ is connected. Again by the upper-semicontinuity theorem, for every point $y$ in $C$ and every $n \geq n_{0}$, since $\left.R^{2} f_{*} F_{n}\right|_{C}=0$, we have [Mu]

$$
\left(R^{1} f_{*} F_{n}\right)_{y} \otimes \mathbb{C} \cong H^{1}\left(X_{y}, F_{n, y}\right) .
$$

For any $m, h^{1}\left(X_{y}, F_{m, y}\right)$ is constant on $B$ since $R^{1} f_{*} F_{m}$ is locally free at every point $y$ on $B$ and $B$ is connected. So for the above $n$ and for all points $y$ in $B$, there is an $l$ such that the map

$$
H^{1}\left(X_{y}, F_{n, y}\right) \longrightarrow H^{1}\left(X_{y}, F_{n+l, y}\right)
$$

is zero. Moreover, for every point $y$ in $C$ and sufficiently large $n$, we have the following commutative diagram:

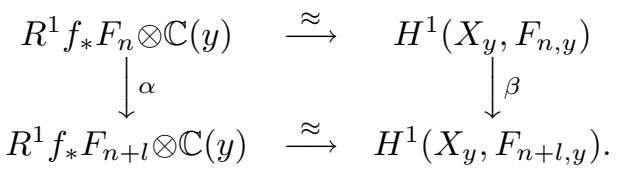

The map $\beta$ is zero for every $y \in B$, so as before, the map

$$
\alpha: \quad\left(R^{1} f_{*} F_{n}\right)_{y} / \mathcal{P}\left(R^{1} f_{*} F_{n}\right)_{y} \longrightarrow\left(R^{1} f_{*} F_{n+l}\right)_{y} / \mathcal{P}\left(R^{1} f_{*} F_{n+1}\right)_{y}
$$

is zero for all points $y$ in $B$. By the local freeness, this says on $B$,

$$
\left.\lim _{\vec{n}} R^{1} f_{*} F_{n}\right|_{B}=0
$$

To see this, fix a point $y_{0}$ in $B$. For any sufficiently large $n$ and for the above $l$, choose an affine open set $V$ containing $y_{0}$ such that both $R^{1} f_{*} F_{n}$ and $R^{1} f_{*} F_{n+l}$ are locally free on $V$. So there are two positive integers $m_{1}$ and $m_{2}$ such that $R^{1} f_{*} F_{n}(V)=\mathcal{O}(V)^{m_{1}}$ and $R^{1} f_{*} F_{n+l}(V)=\mathcal{O}(V)^{m_{2}}$. Now for infinitely many maximal ideals $\mathcal{P}$, we have the commutative diagram

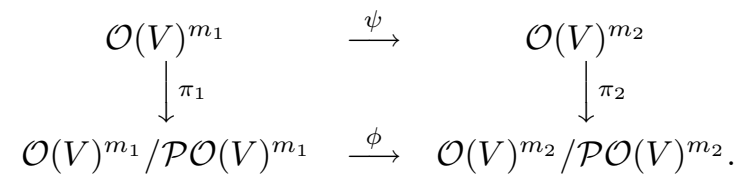


Since $\psi\left(\mathcal{O}(V)^{m_{1}}\right) \subset \cap \mathcal{P O}(V)^{m_{2}}=0$, where $\mathcal{P}$ runs over infinitely many maximal ideals of $\mathcal{O}(V)$, we have $\psi\left(\mathcal{O}(V)^{m_{1}}\right)=0$. This proves

$$
\left.\lim _{\vec{n}} R^{1} f_{*} F_{n}\right|_{B}=0
$$

Since the direct limit of $R^{1} f_{*} F_{n}$ is quasi-coherent, its support is locally closed. Now $B$ is dense and connected in complex topology, so there exists an affine open set $U$ (we come back to Zariski topology!) in $\bar{C}$ such that on $U$, the direct limit

$$
\left.\lim _{\vec{n}} R^{1} f_{*} F_{n}\right|_{U}=0
$$

By Theorem 4 and its remark below, we have proved

Theorem 3. If for every point $y$ in $\bar{C}$ and for every $i>0$,

$$
\lim _{\vec{n}} H^{i}\left(X_{y}, F_{n, y}\right)=0
$$

and for every point $y \in C, D_{y}=X_{y} \cap D$ is a curve on the fibre $X_{y}$, then $\left.R^{2} f_{*} F_{n}\right|_{C}=$ 0 for $n \geq n_{0}$ and

$$
\left.\lim _{\vec{n}} R^{1} f_{*} F_{n}\right|_{U}=0, \quad \text { for a suitable } U .
$$

So $H^{3}\left(Y, \Omega_{Y}^{j}\right)=H^{2}\left(Y, \Omega_{Y}^{j}\right)=H^{1}\left(V,\left.\Omega_{Y}^{j}\right|_{V}\right)=0$ for every $j$, where $V=f^{-1}(U) \cap Y$.

We have seen that $R^{3} f_{*} F_{n}=0$ for every $n$ is determined by the dimension of fibres. Now we explain why for $i=1,2$, if

$$
\lim _{\vec{n}} R^{i} f_{*} F_{n}=0
$$

then $H^{2}\left(Y, \Omega_{Y}^{j}\right)=H^{1}\left(Y, \Omega_{Y}^{j}\right)=0$. For any point $y \in \bar{C}$, choose an affine open set $U$ containing $y$, and let $G$ denote the direct limit of $F_{n}$. Then we have the long exact sequence of local cohomology

$$
\begin{gathered}
H_{Z}^{1}\left(f^{-1}(U), G\right) \longrightarrow H^{1}\left(f^{-1}(U), G\right) \longrightarrow H^{1}\left(f^{-1}(U-\{y\}), G\right) \longrightarrow H_{Z}^{2}\left(f^{-1}(U), G\right) \\
\longrightarrow H^{2}\left(f^{-1}(U), G\right) \longrightarrow H^{2}\left(f^{-1}(U-\{y\}), G\right) \longrightarrow H_{Z}^{3}\left(f^{-1}(U), G\right) \longrightarrow 0,
\end{gathered}
$$

where $Z=f^{-1}(y)$. Since the direct limit commutes with cohomology [H1], for every $i>0$,

and

$$
H^{i}\left(f^{-1}(U), G\right)=\lim _{\vec{n}} H^{i}\left(f^{-1}(U), F_{n}\right)=\lim _{\vec{n}} R^{i} f_{*} F_{n}(U)=0
$$

$$
H^{i}\left(f^{-1}(U-\{y\}), G\right)=\lim _{\vec{n}} H^{i}\left(f^{-1}(U-\{y\}), F_{n}\right)=\lim _{\vec{n}} R^{i} f_{*} F_{n}(U-\{y\})=0,
$$

we have $H_{Z}^{2}\left(f^{-1}(U), G\right)=H_{Z}^{3}\left(f^{-1}(U), G\right)=0$. Let $V=\bar{C}-\{y\}$; from

$$
\longrightarrow H_{Z}^{i}(X, G) \longrightarrow H^{i}(X, G) \longrightarrow H^{i}\left(f^{-1}(V), G\right) \longrightarrow
$$

and for $i=2,3, H_{Z}^{i}(X, G)=H_{Z}^{i}(U, G)=0$. By Lemma 4, we have

$$
H^{2}(X, G)=\lim _{\vec{n}} H^{2}\left(X, F_{n}\right)=0 \Longrightarrow H^{2}\left(Y, \Omega_{Y}^{j}\right)=0
$$

and

$$
H^{3}(X, G)=\lim _{\vec{n}} H^{3}\left(X, F_{n}\right)=0 \Longrightarrow H^{3}\left(Y, \Omega_{Y}^{j}\right)=0 .
$$

Now look at $H^{1}(X, G)$, since

$$
\lim _{\vec{n}} R^{1} f_{*} F_{n}(C)=\lim _{\vec{n}} H^{1}\left(f^{-1}(C), F_{n}\right)=0
$$


and $Y=f^{-1}(C) \cap Y \stackrel{\psi}{\hookrightarrow} f^{-1}(C)$ is an affine morphism ( $D$ is locally defined by one equation), by Grothendieck G], page 100. Finally we get

$$
0=\lim _{\vec{n}} H^{1}\left(f^{-1}(C), F_{n}\right)=H^{1}\left(f^{-1}(C), \psi_{*}\left(\left.F_{n}\right|_{Y}\right)\right)=H^{1}\left(Y, \Omega_{Y}^{j}\right) .
$$

Theorem 4. If

$$
\lim _{\vec{n}} R^{1} f_{*} F_{n}=\lim _{\vec{n}} R^{2} f_{*} F_{n}=0,
$$

or for every $y \in \bar{C}$, if $D_{y}=X_{y} \cap D$ is a curve on the fibre $X_{y}$ and

$$
\lim _{\vec{n}} R^{1} f_{*} F_{n}=\lim _{\vec{n}} H^{2}\left(X_{y}, F_{n, y}\right)=0,
$$

then $H^{i}\left(Y, \Omega_{Y}^{j}\right)=0$ for every $i>0$ and $j \geq 0$.

Remark 4. If $R^{1} f_{*} F_{n}$ are locally free, then $R^{0} f_{*} F_{n}$ and $R^{2} f_{*} F_{n}$ are locally free by $\mathrm{Mu}$, page 50, corollary, and from Theorems 3 and 4, we know that the vanishing direct limit of $H^{i}\left(X_{y}, F_{n, y}\right)$ guarantees the vanishing of the Hodge cohomology of $Y$. So it is almost true that the local vanishing Hodge cohomology on every fibre $S$ over $C$ guarantees the global vanishing of $Y$. The local freeness of $R^{1} f_{*} F_{n}$ also tells us that $H^{i}\left(Y, \Omega_{Y}^{j}\right)=0$ and

$$
\lim _{\vec{n}} R^{1} f_{*} F_{n}=\lim _{\vec{n}} R^{2} f_{*} F_{n}=0
$$

are equivalent.

Remark 5. By Lemma 4, the theorem is true if the above assumptions hold on $C$, i.e.,

$$
\left.\lim _{\vec{n}} R^{1} f_{*} F_{n}\right|_{C}=\left.\lim _{\vec{n}} R^{2} f_{*} F_{n}\right|_{C}=0
$$

or for every $y \in C$,

$$
\left.\lim _{\vec{n}} R^{1} f_{*} F_{n}\right|_{C}=\lim _{\vec{n}} H^{2}\left(X_{y}, F_{n, y}\right)=0 .
$$

Now let us consider the affineness of $Y$. Under the basic assumption (BA), if $Y$ is affine, then every fibre $S$ of $\left.f\right|_{Y}$ over $C$ is affine in the proposition since it is closed in $Y$. Conversely, if every fibre is affine in Theorem 1, is $Y$ affine? In the surface case, it is true. Let us state it precisely. If we have a surjective morphism from a smooth surface $S$ with $H^{i}\left(S, \Omega_{S}^{j}\right)=0$ for every $i>0, j \geq 0$ to an affine curve $C$, then $S$ must be affine. If not, there are nonconstant regular functions on $S$ lifted from regular functions on $C$. But by Lemma 1.8, $[\mathrm{Ku}$, we know there is no such function on $S$. How about the case of threefolds? We can give some answer. By Serre's affineness criterion, $S$ is affine if and only if for all coherent sheaves of ideals $\mathcal{I}_{S}$ on $S, H^{i}\left(S, \mathcal{I}_{S}\right)=0$ for all $i>0$, or if and only if for all coherent sheaves $\mathcal{F}_{S}$ on $S, H^{i}\left(S, \mathcal{F}_{S}\right)=0$. Since the proof of Theorem 2, Theorem 3, and Theorem 4 also works for coherent sheaves, we have

Theorem 5. (1) In the diagram of the proposition, if $Y$ is affine, then every fibre $S$ over $C$ is affine and for every point $y \in \bar{C}$, every $i>0$ and every coherent sheaf $\mathcal{F}$ on $X$,

$$
\lim _{\vec{n}} H^{i}\left(X_{y}, \mathcal{F}_{n, y}\right)=0, \quad \lim _{\vec{n}} R^{2} f_{*} \mathcal{F}_{n}=0
$$


and there is an affine open set $U$ in $\bar{C}$ and an integer $n_{0}$ such that for every $m \geq n_{0}$,

$$
\left.\lim _{\vec{n}} R^{1} f_{*} \mathcal{F}_{n}\right|_{U}=0,\left.\quad R^{2} f_{*} \mathcal{F}_{m}\right|_{U}=0
$$

where $\mathcal{F}_{n}=\mathcal{F} \otimes \mathcal{O}(n D), \mathcal{F}_{n, y}=\left.\mathcal{F}_{n}\right|_{X_{y}}$.

(2) Conversely, if

$$
\left.\lim _{\vec{n}} R^{1} f_{*} \mathcal{F}_{n}\right|_{C}=\left.\lim _{\vec{n}} R^{2} f_{*} \mathcal{F}_{n}\right|_{C}=0,
$$

or for every $y \in C$, if $D_{y}$ is a curve and

$$
\left.\lim _{\vec{n}} R^{1} f_{*} \mathcal{F}_{n}\right|_{C}=\lim _{\vec{n}} H^{2}\left(X_{y}, \mathcal{F}_{n, y}\right)=0
$$

then $Y$ is affine.

Remark 6. If $Y$ is affine, then it is Stein. Theorem 5(2) is also a sufficient condition of Steinness.

Theorem 6. If $H^{i}\left(Y, \Omega_{Y}^{j}\right)=0$ for every $i>0$ and $j \geq 0$, and the $D$-dimension of $X$ is not zero, then $Y$ is affine if and only if for every coherent sheaf $F$ on $X$,

$$
h^{1}\left(X, \lim _{\vec{n}} F \otimes \mathcal{O}(n D)\right)<\infty .
$$

Proof. By the assumption, we know that $Y$ contains no complete curves. By [GH], Proposition 3, we are done.

For the $D$-dimension and Kodaira dimension of $X$, we have

Theorem 7. If $H^{i}\left(Y, \Omega_{Y}^{j}\right)=0$, the D-dimension of $X$ is not zero, and there is a smooth fibre $X_{y_{0}}$ of $f$ over $y_{0} \in \bar{C}$ such that $S_{0}=\left.X_{y_{0}}\right|_{Y}$ is not affine, then the Kodaira dimension of $X$ is $-\infty$ and the D-dimension of $X$ is 1 . Generally, we have

$$
\kappa(\bar{C})+\kappa\left(X_{y_{0}}\right) \leq \kappa(X) \leq \kappa\left(X_{y_{0}}\right)+1 .
$$

In particular, if the genus of $\bar{C}: g(\bar{C}) \geq 2$, then $\kappa(X)=\kappa\left(X_{y_{0}}\right)+1$.

Proof. In the surface case, if $S_{0}$ is not affine and satisfies the same vanishing condition, then the Kodaira dimension of its completion $X_{y_{0}}$ is $-\infty$ and the $D$-dimension is 0 by [I3, [ $\mathrm{Ku}$, Mi]. If $S_{0}$ is not affine, then $X_{y_{0}}$ is birational to either the special ruled surface of case (2) or the special rational ruled surface of case (3) in the first paragraph of Section 0, with $S$ fixed. By deformation theorems of Iitaka [I4, I5], there is an affine open set $U$ in $\bar{C}$ such that every fibre $X_{y}$ of $f$ over $y \in U$ is of the same type. By Theorem 5.11 and Theorem 6.12 of Ueno [U2], we have

$$
\kappa(X) \leq \kappa\left(X_{y_{0}}\right)+1=-\infty .
$$

Combining with the upper semicontinuity theorem, if for the general fibre $X_{y}$ over $y \in \bar{C}, \kappa\left(\left.D\right|_{X_{y_{0}}}, X_{y_{0}}\right)=\kappa\left(\left.D\right|_{X_{y}}, X_{y}\right)$, then

$$
\kappa(D, X) \leq \kappa\left(\left.D\right|_{X_{y_{0}}}, X_{y_{0}}\right)+1 .
$$

Consider $\kappa\left(\left.D\right|_{X_{y_{0}}}, X_{y_{0}}\right)$. If the divisor $D_{y_{0}}=\left.D\right|_{X_{y_{0}}}$ on $X_{y_{0}}$ is a special divisor as $[\mathrm{Ku}]$, i.e., it has no exceptional divisor of the first type and it is a generator of the kernel of the intersection form, then $H^{0}\left(\mathcal{O}_{X_{0}}\left(n D_{y_{0}}\right)\right)=\mathbb{C}$, for every nonnegative integer $n$ (this says $H^{0}\left(\mathcal{O}_{X_{y}}\left(n D_{y}\right)\right)=\mathbb{C}$ for every $n$ and general $y$ ), hence $\kappa\left(\left.D\right|_{X_{y}}, X_{y}\right)=\kappa\left(\left.D\right|_{X_{y_{0}}}, X_{y_{0}}\right)=0, \kappa(D, X)=1$. But we cannot guarantee that $D_{y_{0}}$ 
is such a special divisor. By [I3], properties (1), (2), pages 11 and 12 , let $D_{1}, \ldots, D_{r}$ be prime components of $D$. For all integers $p_{1}, \ldots, p_{r}>0$, we have

$$
\kappa\left(D_{1}+\ldots+D_{r}, X\right)=\kappa\left(p_{1} D_{1}+\ldots+p_{r} D_{r}, X\right)
$$

and if $g: W \rightarrow V$ is a surjective morphism, where $W$ and $V$ are smooth projective varieties and $E$ is an effective divisor on $W$ such that $\operatorname{codim}(g(E)) \geq 2$, then

$$
\kappa\left(g^{*}\left(D^{\prime}\right)+E, W\right)=\kappa\left(D^{\prime}, V\right)
$$

where $D^{\prime}$ is a divisor on $V, g^{*}\left(D^{\prime}\right)=\sum D_{i}^{\prime}$ is the reduced transform of $D^{\prime}$, where $D_{i}^{\prime}$ are irreducible components. By [U2], Lemma 5.3, pages 51 and 52 , if every fibre in the above map $g$ is connected, then we have the $\mathbb{C}$-linear isomorphism

$$
H^{0}\left(V, \mathcal{O}_{V}\left(D^{\prime}\right)\right) \cong H^{0}\left(W, \mathcal{O}_{W}\left(g^{*} D^{\prime}\right)\right) .
$$

From these properties we get the same $D$-dimension. In fact, on the fibre $X_{y}$, the $D_{y}$-dimension does not depend on the support of the divisor $D_{y}$, i.e., $D_{y}$ may contain exceptional curves of the first kind. It also does not depend on the coefficients of the prime divisors of $D_{y}$. In any case, the open part $S=X_{y} \cap Y$ is fixed. Therefore, $\kappa\left(\left.D\right|_{X_{y_{0}}}, X_{y_{0}}\right)=0$. Hence $\kappa(D, X)=1$ if $S_{y_{0}}$ is not affine.

The remaining cases follow from [Ka2], $[\mathrm{V}]$.

Remark 7. In the above proof, if the fibre $X_{y_{0}}=X_{0}$ is smooth, then $S_{0}=X_{0} \cap Y$ is smooth and satisfies $H^{i}\left(S_{0}, \Omega_{S_{0}}^{j}\right)=0$. If $S_{0}$ is not affine, then it is fixed by Mohan Kumar's classification, i.e., it is either a type (2) surface or a type (3) surface (see the first paragraph of Section 0). Here the boundary $D_{0}=X_{0}-S_{0}=\left.D\right|_{X_{0}}$ may not be the special divisor $D_{0}^{\prime}$ in $\mathrm{Ku}$. But by the above argument, $\kappa\left(D_{0}, X_{0}\right)=$ $\kappa\left(D_{0}^{\prime}, X_{0}\right)=0$. It might happen that there is no global divisor $D$ on $X$ such that when restricted to the fibre $X_{0}$, it is the divisor $D_{0}^{\prime}$. Fortunately, we do not need the existence of such a special divisor $D$. In fact, we have $D$ first and then consider its restriction on the fibre.

In the surface case, if $H^{i}\left(S, \Omega_{S}^{j}\right)=0$ for all $i>0$ and $j \geq 0$, and $S$ is not affine, then the Kodaira dimension of its completion is unique and the $D$-dimension is also unique. In the threefold case, is it still true? Using the same notation as in Theorem 7, if $S_{0}$ is affine, then we can choose $U$ such that every fibre $X_{y}$ of $f$ over $U$ has constant Kodaira dimension. If $g(\bar{C}) \geq 2$, then $\kappa(X)=\kappa\left(X_{y}\right)+1$. But under this condition, is $Y$ affine? This is equal to the question that if every (or general) fibre $S$ of $\left.f\right|_{Y}$ over $C$ is affine, is $Y$ affine?

Consider the logarithmic Kodaira dimension $\bar{\kappa}(Y)=\kappa\left(K_{X}+D, X\right)$. For general fibre $X_{y},\left.D\right|_{X_{y}}=D_{y}$ is a divisor on $X_{y}$ with normal crossings [I1]. The logarithmic Kodaira dimension does not depend on the embedding if the boundary is a divisor with normal crossings [Mi]. By Theorem 3, [13], $\bar{\kappa}(Y) \leq \bar{\kappa}(S)+1$, where $S$ is a general fibre. If $S$ is smooth but not affine, then $\bar{\kappa}(S)=-\infty$, therefore $\bar{\kappa}(Y)=-\infty$. Generally, if Iitaka's $\bar{C}_{n}$ conjecture is true [13], i.e., $\bar{\kappa}(Y) \geq \bar{\kappa}(S)+\bar{\kappa}(C)$, then

$$
\bar{\kappa}(S)+\bar{\kappa}(C) \leq \bar{\kappa}(Y) \leq \bar{\kappa}(S)+1 .
$$

In particular, if $\bar{\kappa}(C)=1$, i.e., the genus $g(\bar{C}) \geq 2$, then $\bar{\kappa}(Y)=\bar{\kappa}(S)+1$. In 1978 , Kawamata Ka3] proved this conjecture if the fibre dimension is 1 . 
Finally, we give an example.

Example. Let $S$ be a surface with $H^{i}\left(S, \Omega_{S}^{j}\right)=0$ for all $i>0$ and $j \geq 0$, not affine, and let $C$ be any smooth affine curve. Then $Y=S \times C$ satisfies $H^{i}\left(Y, \Omega_{Y}^{j}\right)=0$ for all $i>0$ and $j \geq 0$ by Künneth's formula (see [Hi] or [SaW]). The $D$-dimension of $X=\bar{S} \times \bar{C}$ is 1 and the Kodaira dimension is $-\infty$ by Theorem 7 . Its logarithmic Kodaira dimension is also $-\infty$. Again by Künneth's formula, if $S$ is of type (3) surface in Mohan Kumar's classification [Ku], then $q(X)=h^{1}\left(\mathcal{O}_{X}\right)=g(\bar{C})$. This example says that $q(X)$ can be any nonnegative integer. So if we choose different $C$ with different genus, the corresponding $X$ are not isomorphic since they have different $q$.

\section{ACKNOWLEDGMENTS}

It is my great pleasure to thank my adviser, Professor N. Mohan Kumar, who led me to the beautiful field of algebraic geometry, in particular, to the fascinating theory of threefolds. I have benefited from communication with the following professors: Michael Artin, Quo-Shin Chi, Steven D. Cutkosky, Xiaojun Huang, Nicholas M. Katz, Yujiro Kawamata, János Kollár, Tie Luo, Noboru Nakayama, Zhenbo Qin, A. Prabhakar Rao, David Wright and Qi Zhang. I would like to thank all of them. I also thank the referee for his/her comments.

\section{REFERENCES}

[Ab] Abhyankar, Shreeram S., Local analytic geometry, Pure and Applied Mathematics, vol. 14, Academic Press, 1964. MR.31:173

[AK] Altman, A.; Kleiman, S., Introduction to Grothendieck Duality Theory, Lecture Notes in Mathematics, Springer-Verlag, 1970. MR 43:224

[Ara] Arapura, D., Complex Algebraic Varieties and their Cohomology, Lecture Notes, 2003.

[Art] Artin, M., Some numerical criteria for contractability of curves on algebraic surfaces. Amer. J. Math. 84(1962), 485-496. MR26:3704

[AtM] Atiyah, M. F. and Macdonald, I. G., Introduction to Commutative Algebra, AddisonWesley, Reading, Mass. (1969). MR39:4129

[B1] Bourbaki, N., Commutative Algebra, Springer-Verlag, 1989. MR90a:13001

[B2] Bourbaki, N., General Topology, Chapters 5-10, Springer-Verlag, 1989. MR90a:54001b

[C] Cutkosky, S. D., Zariski decomposition of divisors on algebraic varieties, Duke Math. J. 53(1986), no. 1, 149-156. Mr87f:14004

[FQ] Friedman, Robert; Qin, Zhenbo On Complex surfaces diffeomorphic to rational surfaces. Invent. Math. 120(1995), no.1, 81-117. MR96d:57032

[G] Grothendieck, A., On the De Rham cohomology of algebraic varieties, Inst. Hautes Etudes Sci. Publ. Math. 29(1966), 95-103. MR 33:7343

[GH] Goodman, J., Hartshorne, R., Schemes with finite-dimensional cohomology groups, American Journal of Mathematics, v. 91, Issue 1, 258-266, 1969. MF 39:2772

[GrH] Griffiths, P. and Harris, J., Principals of Algebraic Geometry, John Wiley \& Sons, Inc., 1994. MR $95 \mathrm{~d}: 14001$

[H1] Hartshorne, R., Algebraic Geometry, Springer-Verlag, 1997.

[H2] Hartshorne, R., Ample Subvarieties of Algebraic Varieties, Lecture Notes in Mathematics, 156, Springer-Verlag, 1970. MR44:211

[H3] Hartshorne, R., Local Cohomology, Lecture Notes in Math., 41, Springer-Verlag, 1967. MR.37:219

[H4] Hartshorne, R., On the De Rham cohomology of algebraic varieties, Publ. Math. IHES 45(1976), 5-99. MR 55:5633

[Hi] Hirzebruch, F., Topological Methods in Algebraic Geometry, Springer-Verlag, 1966. MR 34:2573

[HP] Hodge, W. V. D. and Pedoe, D., Methods of Algebraic Geometry, II, Cambridge University Press, 1952. MR13:972c 
[I1] Iitaka, S., Birational Geometry for Open Varieties, Les Presses de l'Universite de Montreal, 1981. MF,83j:14011

[I2] Iitaka, S., Birational Geometry of Algebraic Varieties, ICM, 1983.

[I3] Iitaka, S., Birational geometry and logarithmic forms, Recent Progress of Algebraic Geometry in Japan, North-Holland Mathematics Studies 73, 1-27. MR.85g:14041

[I4] Iitaka, S., Deformation of compact complex surfaces I, Global Analysis, papers in honor of K. Kodaira, Princeton Univ. Press, 1969, 267-272. MR 40:8086

[I5] Iitaka, S., Deformation of compact complex surfaces II, J. Math. Soc. Japan 22, 1970, 247-261. MR 41:6252

[I6] Iitaka, S., Deformation of compact complex surfaces III, J. Math. Soc. Japan 23, 1971, 692-705. MR 44:7598

[K] Katz, Nicholas M., Nilpotent connection and the monodromy theorem: applications of a result of Turrittin, Publications Mathematiques, 39(1970), 175-232. MR45:271

[Ka1] Kawamata, Y., Characterization of Abelian varieties, Comp. Math. 43(1981), 253-276. MR:83j:14029

[Ka2] Kawamata, Y., Kodaira dimension of algebraic fibre spaces over curves, Invent. Math. 66(1982), 57-71. MR:83h:14025

[Ka3] Kawamata, Y., Addition formula of logarithmic Kodaira dimension for morphisms of relative dimension one. Proc. Internat. Symp. on algebraic geometry at Kyoto (1977), 207-217. Tokyo: Kinokuniya, 1978. MR82d:14019

[Ka4] Kawamata, Y., On the extension problem of pluricanonical forms, Algebraic geometry: Hirzebruch 70 (Warsaw, 1998), 193-207, Contemp. Math., 241. Amer. Math. Soc., Providence, RI, 1999. MR2000i:14053

[Ka5] Kawamata, Y., Deformations of canonical singularities. J. Amer. Math. Soc. 12(1999), no. 1, 85-92. MF 99g:14003

[Kl] Kleiman, S.L., On the vanishing of $H^{n}(X, F)$ for an $n$-dimensional variety, Proceedings of AMS, vol. 18, No. 5, 940-944, 1967. MR.35:4233

[Ko1] Kollár, J., Higher Direct Images of Dualizing Sheaves I, Ann. of Math., v.123, 1(1986), 11-42. MR.87c:14038

[Ko2] Kollár, J., Higher Direct Images of Dualizing Sheaves, II, Ann. of Math., v.124, 1(1986), 171-202. MR87k:14014

[KM] Kollár, J., Mori, S., Birational Geometry of Algebraic Varieties, Cambridge University Press, 1998. MR2000b:14018

[Ku] Kumar, N. Mohan, Affine-Like Surfaces, Journal of Algebraic Geometry, 2(1993), 689-703. MR 94i:14039

[KuM] Kumar, N. Mohan; Murthy, M. Pavaman Algebraic cycles and vector bundles over affine threefolds, Ann. of Math. (2), no. 3, 579-591. MR84d:14006

[L1] Luo, Tie, Global 2-forms on regular 3-folds of general type, Duke Math. J. 71 (1993), no.3, 859-869. MR94k:14032

[L2] Luo, Tie, Global holomorphic forms 2-forms and pluricanonical systems on threefolds, Math. Ann. 318 (2000), no. 4, 707-730. MR2002a:14043

[LZ] Luo, Tie; Zhang, Qi, Holomorphic forms on threefolds, preprint, 2003.

[M] Matsuki, Kenji, Introduction to the Mori program, Universitext. Springer-Verlag, New York, 2002. MR.2002m:14011

[Ma] Matsumura, H., Commutative Algebra, Second edition, W. A. Benjamin Co., New York, 1980. MR $82 \mathrm{i}: 13003$

[Mi] Miyanishi, M., Non-complete Algebraic Surfaces, Lecture Notes in Mathematics, 857, Springer-Verlag, 1981. MR83b:14011

[Mo1] Mori, S., Birational Classification of Algebraic Threefolds, ICM, 1990. MR92m:14043

[Mo2] Mori, S., Birational Classification of Algebraic Threefolds, Algebraic geometry and related topics (Inchon, 1992), 1-17, Conf. Proc. Lecture Notes Algebraic Geom., I, Internat. Press, Cambridge, MA, 1993. MR95f:14026

[Mu] Mumford, D., Abelian Varieties, Oxford University Press, 1970. MR 44:219

[N] Nagata, M., Imbedding of an abstract variety in a complete variety, J. Math. Kyoto Univ. 2(1962), 1-10. MR26:118

[Na1] Nakayama, N., Invariance of the plurigenera of algebraic varieties, Topology 25 (1986), 237-251. MR87g:14034

[Na2] Nakayama, N., Zariski decomposition and abundance, RIMS preprint (June 1997). 
[Na3] Nakayama, N., Invariance of the plurigenera of algebraic varieties, RIMS preprint (March 1998).

[NS] Norguet, F.; Siu, Y.T. Holomorphic convexity of spaces of analytic cycles. Bull. Soc. Math. France 105, 191-223(1977). MR.58:28677

[P] Peternell, T., Hodge-kohomolodie und Steinsche Mannigfaltigkeiten, Complex Analysis, Aspects of Mathematics, Vieweg-Verlag, 1990, 235-246. MR92j:32038

[SaW] Sampson, J. H., Washnitzer, G., A Künneth formula for coherent algebraic sheaves, Illinois J. Math. 3, 389-402(1959). MR21:5636

[Se] Serre, J. P., Quelques problèmes globaux relatifs aus variétés deStein, Collected Papers, Vol.1, Springer-Verlag(1985), 259-270.

[Sh] Shafarevich, I. R., Basic Algebraic Geometry 1, 2, Springer-Verlag, 1994.

[Sho1] Shokurov, V. V., 3-fold log flips. Izv. Russ. A. N. Ser. Mat., 56: 105-203, 1992. MR 93j:14012

[Sho2] Shokurov, V. V., 3-fold log models. Algebraic geometry, 4. J. Math. Sci. 81(1996), no. 3, 2667-2699. MR97i:14015

[Si1] Siu, Y.-T., Analytic sheaf cohomology of dimension $n$ of $n$-dimensional complex spaces. Trans. Amer. Math. Soc. 143, 77-94(1969). MR 40:5902

[Si2] Siu, Y.-T., Invariance of plurigenera, Invent. Math. 134, 661-673(1998). MR99i:32035

[U1] Ueno, K., Algebraic Geometry 1, 2, AMS, 1999. MR2000g:14001 MR2001j:14001

[U2] Ueno, K., Classification Theory of Algebraic Varieties and Compact Complex Spaces, Lecture Notes in Mathematics, v.439, 1975, Springer-Verlag. MR58:22062

[V] Viehweg, E., Weak positivity and the additivity of the Kodaira dimension certain fibre spaces, Adv. Studies Pure Math. 1(1983), 329-353. MR85b:14041

[Z] Zariski, O., The theorem of Riemann-Roch for high multiples of an effective divisor on an algebraic surface, Ann. of Math. (2), 76(1962), 560-616. MR25:5065

[Zh] Zhang, Qi, Global holomorphic one-forms on projective manifolds with ample canonical bundles, J. Algebraic Geometry 6 (1997), 777-787. MF99k:14071

Department of Mathematics, Washington University, St. Louis, Missouri 63130

E-mail address: zhj@math.wustl.edu

Current address: Department of Mathematics, University of Missouri-Columbia, Columbia, Missouri 65211 